\title{
The COVID PIVOT - Re-orienting Child and Youth Mental Health Care in the Light of Pandemic Restrictions
}

\author{
Liza Hopkins $^{1}$ (D) . Glenda Pedwell ${ }^{1}$
}

Accepted: 28 February 2021 / Published online: 22 March 2021

(c) Crown 2021

\begin{abstract}
The COVID pandemic required significant changes in the provision of youth mental health services during the period of lockdown/stay at home orders. Things which were identified as changing significantly during this period included: service via telehealth; working from home, split teams (to reduce infection risk), and social (physical) distancing. An online survey of clinicians was conducted involving both closed and open ended questions. Service staff identified significant benefits from the changes to the way services were delivered as well as some impediments and challenges. Advantages in the new way of working revolved around the flexibility of the virtual service, with appointments online enabling families to more easily overcome issues of transport, work commitments, childcare and disruption to routines and timing. The online platforms also enabled some family members to participate who otherwise might not have been able to come to appointments in person. Disadvantages included where there were issues with availability and access to appropriate technology or private spaces, or when the young person was very young, very unwell, unstable, isolated or at higher risk. This study suggests that telehealth and flexible working arrangements have become an essential new element in the clinicians' toolkit to be offered either alone or as a supplement to in person interventions.
\end{abstract}

Keywords Service re-orientation · Telehealth - Telepsychiatry · Youth mental health · Flexible work arrangements

\section{Introduction}

The shift in the provision of ambulatory mental health care to infants, children, young people and their families driven by the global COVID-19 pandemic has arguably been the most dramatic change in the history of mental health care [1]. Community based mental health care had traditionally been delivered in a clinic, with limited home based outreach and a few forays into telepsychiatry in remote areas. While provision of mental health care via online modalities has been emerging over the past 10 years, concerns related to

Liza Hopkins

1.hopkins@alfred.org.au

1 Alfred Health, Child and Youth Mental Health Service, 999 Nepean Hwy, Moorabbin, VIC 3189, Australia 
authenticity offered by in person contact, privacy, security, access and risk [2-6] arguably restricted mainstream take up of these modalities. The hard lockdown associated with national public health responses to the pandemic occasioned a concomitant shift of service delivery from face to face to remote modalities and a requirement for many staff to work from home [6]. These shifts, which might have taken decades to occur under ordinary circumstances, were achieved within a matter of weeks.

This rapid transition, however, while essential in the pandemic context, couldn't occur at the cost of quality and safety [7]. Research into the delivery of mental health care online has previously identified issues such as the ability of clinicians to establish the therapeutic alliance, the feasibility of access for particular patient groups and technological phobia of clinicians, as well as the potential to further marginalise those without access to technology or ability to use it $[3,8]$. Other concerns include the suitability of telehealth for acute presentations, or for first appointments with new clients [9]. Nonetheless, one of the few studies to examine the effectiveness of synchronous telehealth compared to face to face services in reducing depression symptoms found no significant difference in psychotherapy delivered between the two modalities [4]. In addition, studies have shown that people with serious mental illness are open to receiving mental health care via digital means, and that this is especially true for young people [10].

In addition to clinical effectiveness and accessibility, there were policy and financing constraints on delivery of telehealth which inhibited change in practice until the pandemic crisis. Governments were forced to reconsider and rapidly revise concepts of mental health service delivery and soon recognised that reimbursement of telehealth services could offer an acceptable alternative to face to face services [11]. Thus the scene was set for a pivot of service delivery in child and youth mental health which has the potential to alter forever the way services engage with mental health clients [12].

In light of this pivot, a project was developed to assess the nature and impact of these changes for three child and youth mental health services in southeastern Melbourne. The three services are part of a larger public mental and addiction health service, and comprise a primary care, walk in clinic for young people aged $12-25$, a tertiary child and youth mental health service (ages 0-25), and a youth Early Psychosis service (also ages 12-25).

Youth mental health care was considered an essential service under the lockdown (or shelter in place) arrangements, however to assist with social distancing and reduce potential spread of the virus, these services moved rapidly to using telehealth. The split team model for on and off site staffing saw staff allocated to one of two sub teams alternating one week in the clinic and one week at home. In this paper we consider the findings of this project across three domains: the benefits and challenges of delivering service via telehealth; the benefits and challenges of mental health staff working from home; and implications for future service delivery.

\section{Method}

The project involved an online survey of staff across the three services undertaken during the first period (April-May) of lockdown during the 2020 COVID pandemic. The staff survey was sent to 199 staff members working across the three identified child and youth mental health services. 113 responses were returned, giving a participation rate of $57 \%$.

Of these, 10 were staff of the primary service, 39 were staff of the tertiary services, 26 were staff of the Early Psychosis service, 24 were in administration or management across 
the three services, and 21 were members of the peer and recovery teams (seven respondents indicated that they worked across more than one of these teams).

\section{Results}

The shift from in person to provision of service via telehealth happened very suddenly in mid-March 2020. The following tables shows the increase from no telehealth in early March to frequent use by the child and youth services by mid-April (Fig. 1).

When compared with other areas of Mental and Addiction Health, it will be seen that the three youth services were rapid and early adopters of the shift to telehealth (Fig. 2).

\section{Working from Home}

The survey asked whether the respondent's way of working had changed due to COVID$19.96 \%$ of respondents $(n=109)$ agreed that their way of working had changed during this time, including:

- More use of telehealth

- More use of telephone

- Shift of internal and external meetings to online

- Splitting and rotating the roster of teams

Respondents were also offered the opportunity to suggest other ways in which ways of working had changed. Suggestions included:

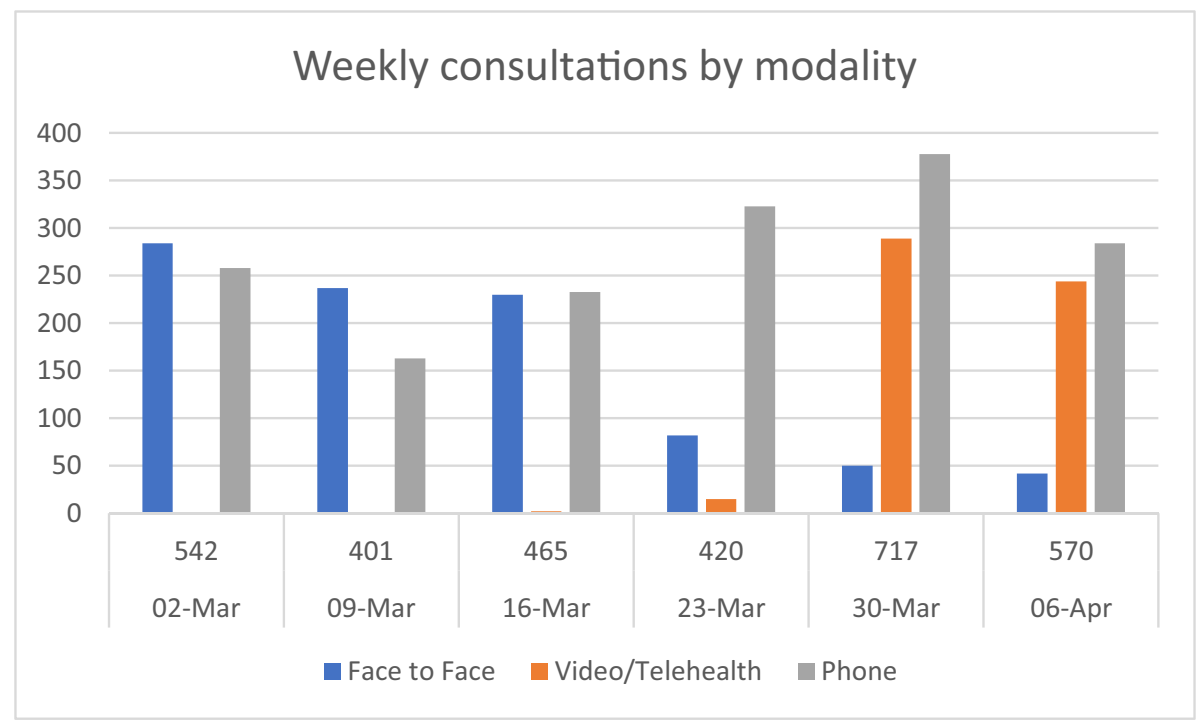

Fig. 1 Weekly consultations by modality 


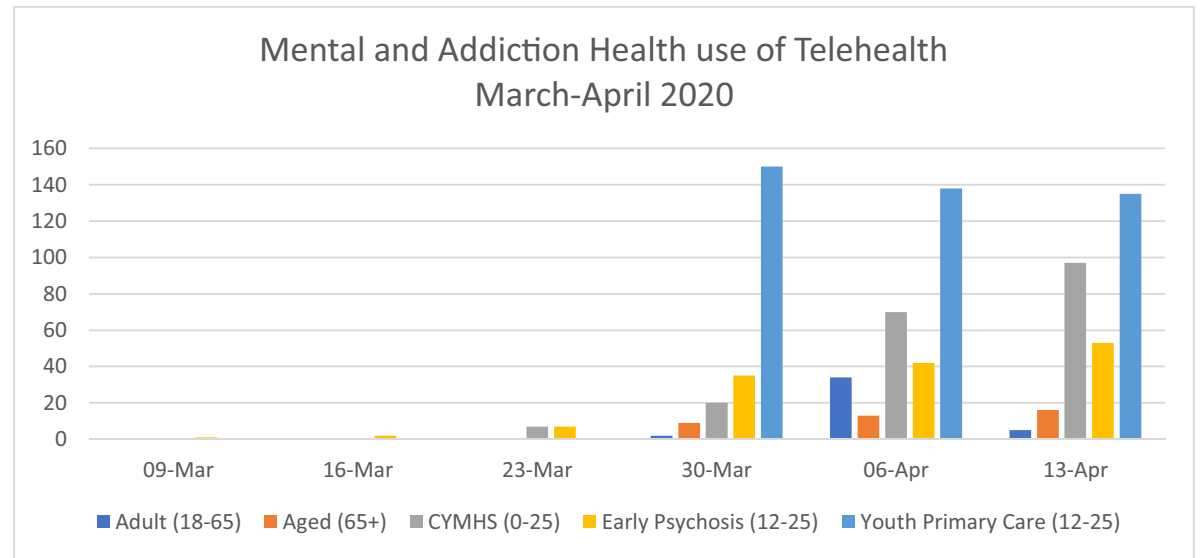

Fig. 2 Mental and Addiction Health use of Telehealth, March-April 2020

I find my administration burden has been overall decreased but some tasks have been made more difficult. For instance remotely I only have access to [some shared drives].... But my home internet connection is far superior to the clinic so I have had NO issues with any telehealth. I haven't had any DNAs [Did Not Attend]. Overall, it's been fantastic.

The percentage of time spent working at home is given in the table below (Fig. 3). Respondents were asked to what extent they were able to access and manage things that they needed in order to work from home (Fig. 4).

\section{Over the past month have you worked from home?}

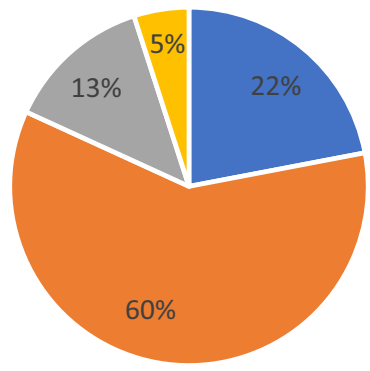

Most of the time $\quad$ Half of the time $\quad$ Some of the time Not at all

Fig. 3 Staff time spent working at home, April 2020 


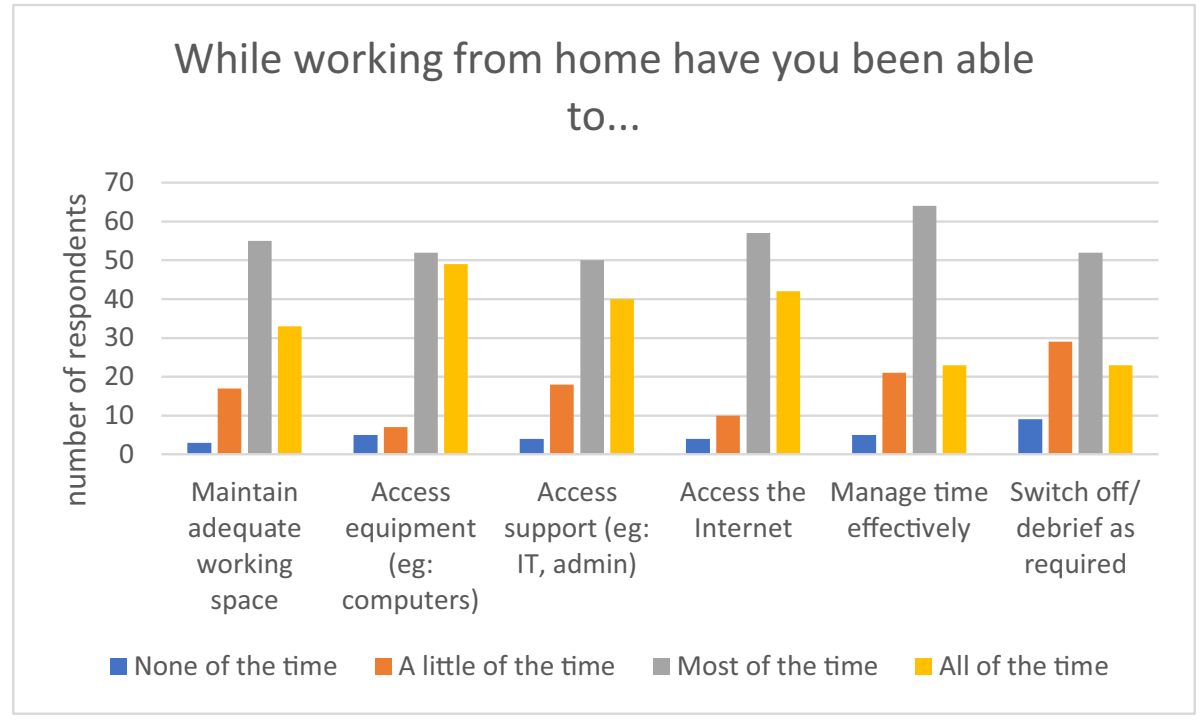

Fig. 4 Ability to access necessities and manage while working from home

This chart demonstrates that most staff were able to manage their working from home requirements most of the time. The hardest thing to manage was the ability to switch off or debrief after work. Approximately one third of staff were able to do this not at all or only a little.

Staff further identified numerous benefits of work from home. These could be grouped into six main themes:

- Autonomy

- Enjoying the home physical environment

- Reduced logistics of transport, child care etc.

- Ability to prioritise clinical work in the clinic and report writing at home

- Advantages of telehealth, and

- COVID specific responses (such as reduced need for hygiene practices such as hand washing/sanitising at home).

A typical comment included:

Open plan office space is often too noisy and disruptive to complete work to a good standard. Working from home allows for allocated tasks to be focused on without interruptions. This also applies to phone calls with clients and external agencies.

Another benefit was the ability to separate the kinds of work completed at home compared with the kinds of work undertaken in the clinic. Examples of these responses included:

Less face-to-face/direct client work at home allows for weeks at the clinic to be more focused on clinical tasks (intake, appointments) which is very busy, and 
week from home I am able to complete admin tasks, engage in supervision, secondary consults, as well as usual team and leadership meetings.

In addition to these benefits, staff also identified numerous challenges while working from home. These could be grouped under the following headings:

- Juggling work and home responsibilities, including managing boundaries

- Isolation/ lack of collegial support

- Access to equipment

- Managing the home environment

- Communication.

An example of these challenges included:

The speed of the transition was quite confronting. In the first week there was a lots of logistical and professional boundaries work to be navigated.

Staff were asked what support and resources they had found to be necessary when working from home. Contact with colleagues topped the list, with almost one third of respondents (36/113) reporting this to be something they needed and wanted. Typical responses included:

As I mainly work on a computer, I have everything I need, except face to face interaction.

The second most commonly reported need was for adequate IT equipment (21 responses/ 113), with an additional 10 respondents reporting the need for adequate IT support. Examples of responses included:

[Service] laptop or tablet is needed to connect to [service] systems remotely. Logging into remote access from a private computer is difficult and cumbersome.

A further 19 respondents identified internet access as an important consideration when working from home:

Excellent internet [is required]. Better connection between remote connector and medical software. [Software] has not worked well enough-it's slow and connection is hard to make and then keeps dropping out. This has really slowed me down.

Twenty-one respondents identified their physical space and access to privacy as important resources, while an additional six respondents reported the need to establish clear boundaries between work and home. Staff were also asked what they had liked about working from the clinic during this period. By far the most common response to this question was social connection (44 responses), such as:

SEEING PEOPLE! Being physically out of my home space and able to switch off at the end of the day.

Respondents also reported that they appreciated having access to workplace infrastructure, and supports while physically in the office.

I am more efficient at work because the systems are more efficient and work cohesively. I need my stand up desk, ergo[nomic] chair, head set for phone and natural light (work station is beside window). I can debrief and feel connected to colleagues. 
Only $11 \%$ of respondents noted that they would not like to work at home in the future, while an even smaller number (2\%) wanted to work at home all the time. The majority of staff would like a degree of flexibility in working between home and the clinic, which would represent a significant change in the way the service is delivered going forward (Fig. 5).

\section{Telehealth}

Despite a degree of anxiety which was noted prior to the roll out of telehealth, after using the online system for a few weeks, less than $10 \%$ of respondents reported that they never liked using it and would never like to use it in the future (Fig. 6).

There were several advantages of using telehealth identified, including:

- Reduced travel and logistical difficulties for young people and their families

- The inclusion of family members who may not otherwise be able to attend

- Being able to see the young person in their own setting, and observe their living arrangements

Staff were asked to consider which clients benefit most from telehealth. Responses included:

- Teenagers and young adults

- Parents

- Those with good access to technology

- Those with anxiety

\section{I would like to work from home in the future}

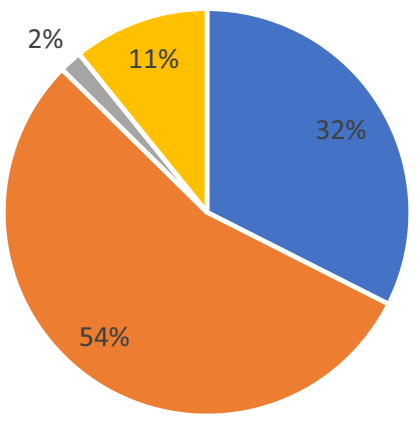

- A bit of the time $\quad$ Some of the time $\quad$ All of the time Not at all

Fig. 5 Staff desire to continue working from home 


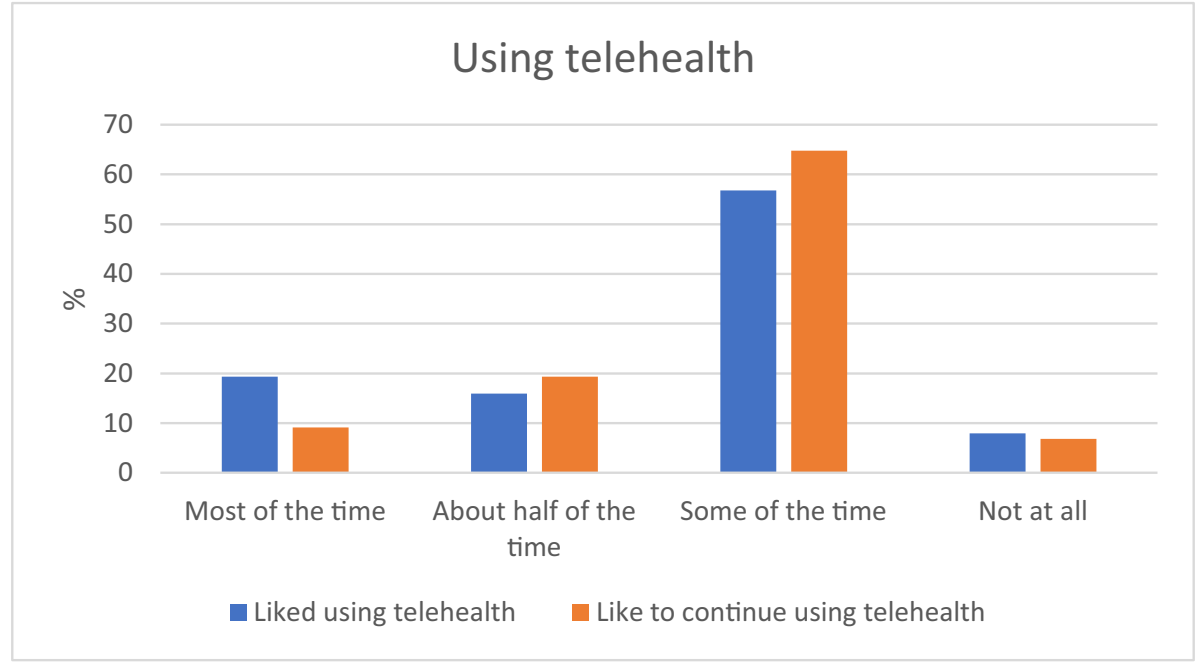

Fig. 6 Staff desire to use and continue using telehealth

- People who may have difficulties with transport or who live a long way away from the clinic sites

- Those who are already well engaged with the service and who are stable in their presentations

- Children and young people with Autism Spectrum Disorder, Attention Deficit Hyperactivity Disorder and mild Intellectual Disability, and

- Clients who are socially isolated (either for COVID related or other reasons).

Typical responses as to who benefits most included:

White, middle class parents who are professionals and have some experience with technology. In addition, it is most suitable for clients with whom you have had a relationship / met before COVID.

Kids love it because it's what they are used to and they are far more comfortable with it. I've seen so many different sides to my patients since the combination of in person and telehealth. Those kids who also have sensory processing issues really benefit by not having to travel to the clinic, be exposed to the clinic and the disruptions that occur to their routine.

There were also several challenges in using telehealth identified, the greatest of which was technological issues, such as low bandwidth, lack of access to hardware and lack of knowledge about how to use the system. Lack of engagement and reduced rapport were also common challenges.

Other more specific concerns included:

- Clients joining telehealth seeking support without appointments

- Initial appointment may be harder by telehealth

- Hard when young person wants telephone only service and there are risk concerns

- Reduced ability to track non-verbal communication 
- Telehealth is not appropriate for all psychopathologies such as those who are anxious/ avoidant/have personality disorders

- Child unable to sit at screen due to attention/behavioural difficulties.

The survey then asked about staff perceptions of which clients receive the least benefits from the telehealth service. Responses included:

- Very young children (pre-school)

- The technologically disadvantaged

- Those with specific diagnoses (such as Borderline Personality Disorder and Eating Disorders) or who are more unwell or unstable

- People presenting for new or initial appointments, or for assessment

- Young people who are poorly engaged or considered to be high risk

- Young people who need the social contact of a face to face service.

Typical responses included:

It is not possible for parents to get their baby or child on screen and for us to observe and reflect on the interactions (ie our core work), beyond brief moments, so we have had to rely on mainly parent work: only part of what we [normally] offer.

Clients from lower socio-economic groups, with less experience with technology. These clients have generally not had the most up to date operating system to support telehealth, so appointments need to be provided via a telephone call. These clients have also been more inclined to not take up the offer of a telehealth appointment, potentially because of a reluctance to have us see them in their house.

When asked what training helped them to use telehealth, 30\% reported that formal training was most helpful while $20 \%$ found informal training, practice and "trial and error" to be most useful.

The final question on the survey provided an open-ended opportunity to comment on the changes in the workplace due to pandemic restrictions. Typical additional comments around the advantages and disadvantages of working from home included:

In the future it would be great to have the opportunity to work from home as it has definitely had its benefits for clients/families and clinicians. Personally, mentally I have been a lot happier in life and in work.

Such benefits have important implications for wellbeing, workplace burnout and staff turnover.

Typical comments around the advantages and disadvantages of using telehealth included:

I think it would be regressive to return to 'business as usual'. There are so many benefits to telehealth and working from home-for patients and workers alike. We can do it, it does work, it's great for clients, it's great for employees, it's great for the environment and it allows us to deliver services in a flexible and truly patient centred way, that also enable employees to have fulfilling and flexible lives that don't revolve around a nineteenth century paradigm of office based 9-5 pm work. 
Comments on the management of the changes focussed on the ways in which the three services were able to negotiate the changes in work practices while still providing a high level of care to young people. Comments included:

It has been a hard journey for the service \& it is pretty amazing how everyone has passionately pivoted. The biggest piece of feedback from Young People is that we are now meeting them where they are at with these spaces rather than making them meet us where we are at.

Additional comments which addressed staff well being included:

Normally in the drive home from work you can switch off the day, difficult to do in this environment and so separation between work and home becomes difficult.

One final additional comment summed up many of the themes from the entire survey:

I'm open to some home and online working but there's so much in the human/ emotional/ relational/ physiological landscape that in my opinion cannot be effectively or appropriately replicated online.

\section{Discussion/Conclusion}

The disruptions caused by the emergence of the novel coronavirus in 2020 were felt across all aspects of most people's work and personal lives. Mental health services were no exception, and there were numerous challenges involved in pivoting the service from business as usual to a socially distanced model. These challenges, as well as some associated benefits, were felt by all teams in the services, including clinical and non-clinical staff, as well as by children, young people and families receiving services.

The three youth mental health services which took part in this study each have different individual characteristics, such as client age, client presentations/ diagnoses and therapeutic approaches. Nonetheless, staff across all services, with the support of management, were able to quickly adjust to providing services in a wholly new way. Significant change during this period included: service via telehealth, social (physical) distancing, working from home and split teams. The change occurred very abruptly, and with little warning. Despite this, there were numerous benefits to both staff and young people/ families, as well as the inevitable challenges [2].

This project has shed initial light on the experience of staff in shifting service provision in response to the COVID pandemic and revealed some of the advantages and disadvantages of providing child and youth mental health services using telehealth platforms. Much work remains to be done in this space [13]. Future work could focus more closely on areas such as:

- The nature of the telehealth experience

- Child, young person and family perspectives on the benefits and challenges of telehealth

- Changes in demand for service and the nature of mental health presentations during a national emergency. 
The findings of this study may be useful in informing service change going forward, both in the event of the sudden discovery of a cure/globally-effective vaccine for COVID19 resulting in a return to business as usual or (more likely) in the event of ongoing pandemic repercussions, and a re-orientation of services to meet ongoing changes in need and opportunity.

Acknowledgements We acknowledge and thank the participants who gave up their time to contribute to the research process. This work was carried out on the traditional lands of the people of the Kulin nation and we pay our respects to Elders past, present and emerging. Both authors were employed by the institution within which the research took place.

Authors' Contributions Both authors contributed to the design and conduct of the research and to the writing of this paper.

\section{Data Availability N/A}

Code Availability N/A

\section{Declarations}

Ethics Approval The research was carried out with ethical approval from Alfred Health Human Research Ethics Committee (project \# 279/20).

Consent to Participate Informed consent was obtained from all individual participants included in the study.

Consent for Publication Informed consent was obtained from all individual participants included in the study.

Competing Interests The authors declare they have no competing financial or commercial interests.

\section{References}

1. Pinals DA, Hepburn B, Parks L, Stephenson AH. The behavioral health system and its response to COVID-19: a snapshot perspective. Psychiatr Serv Adv. 2020. https://doi.org/10.1176/appi.ps. 202000264.

2. Moreno C, Wykes T, Galderisi S, et al. How mental health care should change as a consequence of the COVID-19 pandemic. Lancet Psychiatry. 2020. https://doi.org/10.1016/S2215-0366(20) 30307-2.

3. Lal S, Adair C. E-mental health: a rapid review of the literature. Psychiatr Serv. 2014;65(1):24-32. https://doi.org/10.1176/appi.ps.201300009.

4. Osenbach JE, O'Brien KM, Mishkind M, et al. Synchronous telehealth technologies in psychotherapy for depression: a meta-analysis. Depress Anxiety. 2013;30:1058-67.

5. Hosie A, Vogl G, Carden J, Hoddinott J, Lim S. A way forward: equipping Australia's mental health system for the next generation. 2015. Reach Out Australia and EY. https://www.semanticscholar.org/ paper/A-way-forward\%3A-equipping-Australia's-mental-health-Hosie-Vogl/35d6fb4cde8961f33b9a 3c93f4e521dece5056cc. Accessed 18 Mar 2021.

6. Ramtekkar U, Bridge JA, Thomas G, et al. Pediatric telebehavioral health: a transformational shift in care delivery in the era of COVID-19. J Internet Metal Health 2020; 7(9): e20157. https://doi.org/10. 2196/20157.

7. Beidas R, Wiltsey-Stirman S. Realizing the promise of learning organizations to transform mental health care: telepsychiatry care as an exemplar. Psychiatr Serv Adv. 2020. https://doi.org/10.1176/appi. ps.202000257.

8. Reay RE, Looi JC, Keightley P. Telehealth mental health services during COVID-19: summary of evidence and clinical practice. Australas Psychiatry. 2020. https://doi.org/10.1177/1039856220943032.

9. Looi J, Pring W. Private metropolitan telepsychiatry in Australia during Covid-19: current practice and future developments. Australas Psychiatry. 2020. https://doi.org/10.1177/1039856220930675. 
10. Brunette MF, Achtyes E, Pratt S, Stilwell K, Opperman M, Guarino S, Kay-Lambkin F. Use of smartphones, computers and social media among people with SMI: opportunity for intervention. Community Ment Health J. 2019;55:973-8. https://doi.org/10.1007/s10597-019-00431-7.

11. Smith T, Sullivan A-MT, Druss BG. Re-designing public mental health systems post-COVID-19 Psychiatric Services in Advance. 2020. https://doi.org/10.1176/appi.ps.202000400.

12. Vogt EL, Mahmoud H, Elhaj O. Telepsychiatry: implications for psychiatrist burnout and well-being. Psychiatr Serv. 2019;70(5):422-4. https://doi.org/10.1176/appi.ps.201800465.

13. Smith K, Ostinelli E, Macdonald O, Cipriani A. COVID-19 and telepsychiatry: development of evidence-based guidance for clinicians. JMIR Ment Health 2020;7(8):e21108. https://doi.org/10.2196/ 21108.

Publisher's Note Springer Nature remains neutral with regard to jurisdictional claims in published maps and institutional affiliations.

Liza Hopkins is an Evaluation and Research Development Officer with Alfred Health. She holds a PhD and is experienced in developing and conducting research and evaluation projects across a range of fields including youth, health, mental health, education, communications and social inclusion.

Glenda Pedwell is the Deputy Director of Alfred Mental and Addiction Health: Allied Health, Child Youth and headspace. She holds a BachOT and GradDipPubPol and is a specialist in child and youth mental health. 